\title{
Strategies to promote regular exercise in adolescent and young adult cancer survivors
}

This article was published in the following Dove Press journal:

Clinical Oncology in Adolescents and Young Adults

6 November 2015

Number of times this article has been viewed

\section{Margaux J Barnes \\ Krista Casazza \\ Heather Austin}

Department of Pediatrics, Division of General Pediatrics and Adolescent Medicine, University of Alabama at Birmingham (UAB), Birmingham, AL, USA
Correspondence: Margaux J Barnes Department of Pediatrics, Division of General Pediatrics and Adolescent Medicine, University of Alabama at Birmingham (UAB), CPPI 3I0, 1600 7th Ave S, Birmingham, AL 35233, USA

Tel +l 2056385382

$\mathrm{Fax}+\mathrm{I} 2059756503$

Email mbarnes@peds.uab.edu

\begin{abstract}
Adolescents and young adults (AYAs) diagnosed with cancer are at increased risk for current and future health problems and premature death. Given the known risks for increased mortality and morbidity, fostering the development of exercise behaviors that may target the specific late effects experienced by AYA cancer survivors is crucial for healthy aging. Exercise is particularly important in the mitigation of late effects as it alters metabolic pathways that ultimately influence the development of chronic diseases associated with cancer treatment in AYAs. Despite the importance of exercise, a large proportion of AYA survivors do not meet recommended guidelines for exercise. The current review summarizes the beneficial effects of exercise in AYA survivors, evaluates strategies utilized in interventions that have been developed to address exercise engagement, and provides recommendations for psychosocial strategies on how to improve these behaviors in this population. A total of nine empirical articles examining exercise interventions in AYAs with a history of cancer were included for review. While several exercise strategies appear promising, more research is needed to evaluate long-term influence of strategies on exercise engagement, as well as the interactions these strategies have on AYA exercise maintenance and health outcomes as they continue into adulthood. Future interventions should evaluate key psychosocial strategies to increase both short- and long-term exercise adherence as well as overall health benefits.
\end{abstract}

Keywords: oncology, physical activity, adolescents, young adults

\section{Introduction}

In the USA, an estimated 26,000 adolescents and young adults (AYAs) between the ages of 15 and 29 years are diagnosed with cancer each year. ${ }^{1}$ While advances in medical treatments have led to increased survival rates upward of $80 \%$ in AYAs, cancer and its associated treatment increases risk for long-term morbidity and mortality. ${ }^{2}$ The mortality rate in AYA survivors due to cancer recurrence, secondary cancers, and cardiovascular and lung disease as a result of their original diagnosis and subsequent treatment greatly surpasses rates seen in the general population and survivors of cancer diagnosed later in life. ${ }^{3-5}$ AYA cancer survivors also report a significantly higher prevalence of adverse physiologic and psychosocial outcomes including obesity, cardiovascular disease, hypertension, diabetes, osteoporosis, asthma, disability, and mood issues compared to peers without a history of cancer and subsequently increased risk of morbidity and mortality. 6,7

Given the known risks for increased mortality and morbidity, fostering the development of healthy lifestyle behaviors that may target specific late effects early in life is crucial for healthy aging in AYAs. Exercise is particularly important in the 
mitigation of late effects as it alters metabolic pathways that ultimately influence the development of chronic diseases associated with cancer treatment. In both the general population and cancer survivors specifically, the benefits of engagement in regular exercise is well documented and includes (but is not limited to) increased cardiorespiratory fitness, maintenance of muscle and bone strength, reduced anxiety and depression, reduced risk of some future cancers, and more favorable cardiovascular and metabolic profiles. $^{8-14}$

Despite the increased need for adequate exercise, a significant proportion of AYAs with a history of cancer do not meet the Centers for Disease Control and Prevention (CDC)'s recommended weekly minimum of 150 minutes of moderateto-vigorous-intensity exercise. ${ }^{9,15-17}$ It is estimated that $48 \%-65 \%$ of AYA survivors are currently not meeting these recommendations, regardless of cancer diagnosis. ${ }^{14,18,19}$ The only study identified to document patterns of exercise engagement in AYA survivors across the cancer experience found that while $70 \%$ of AYAs in the sample met CDC recommendations prior to treatment, only $10 \%$ reported meeting guidelines during treatment, and less than $49 \%$ reported meeting exercise guidelines as survivors. ${ }^{19}$ Even though there was an increase in survivors who reported adequate exercise after treatment compared to during treatment, the total time spent exercising remained significantly lower than pre-diagnosis activity. ${ }^{19}$ While rates of inactivity are similar to those seen in healthy AYAs, the lack of adequate exercise in the AYA survivor population has significant clinical importance and may contribute to survivors' already heightened risk for increased morbidity and mortality. ${ }^{20}$

Substantial evidence in animals and humans highlights the detrimental effects of physical inactivity across systems emanating from poor musculoskeletal development in "healthy" states that would undoubtedly be augmented in the proinflammatory environment of cancer treatment. For example, just 1-3 weeks of bed rest in otherwise healthy, active young men had a more profound impact on physical work capacity than did 3 decades of aging in the same men. ${ }^{21-23}$ Within days, decreased muscle activity due to prolonged bed rest led to decreased skeletal muscle insulin sensitivity, insulin signaling, fitness, leg muscle mass, and increased intraabdominal fat. ${ }^{24}$ Impaired insulin signaling, altered glucose and lipid metabolism, and increased central adiposity were also noted immediate effects of bed rest. ${ }^{25-27}$ While bed rest is an extreme model of inactivity and does not accurately mimic low levels of physical activity seen in AYA survivors, the findings are germane to AYA survivors and the potential musculoskeletal and metabolic late effects they may have accrued as a result of their diagnosis and treatment. ${ }^{28}$ Specific to young adult survivors of cancer, research indicates that AYAs show accelerated aging, poorer physical fitness and muscle function, and decreased bone mineralization, related to their disease and treatment as well as inadequate physical activity. ${ }^{29-32}$ The manifestations of many of the comorbidities observed in AYA have been directly linked to the aforementioned decline in musculoskeletal function. Central to this principle is the availability of nutrients across systems to permit necessary biological adaptations catalyzed by endocrine and paracrine effects of muscle contraction. As musculoskeletal function is among the greatest determinants of morbidity and mortality across the life course, and the strength/structural properties of the musculoskeletal system are largely established in adolescence and young adulthood, perturbations during this time may have profound implications as AYAs continue to age.

Given the crucial role of exercise in the mitigation of survivors' long-term health and well-being, the current review aims to evaluate the literature addressing strategies used to promote regular exercise in AYAs with a history of cancer. Based on this review, we provide recommendations for specific psychosocial strategies to promote exercise engagement with consideration of developmental issues relevant to AYA survivors. Given the relatively small body of literature addressing AYA exercise strategies, supportive evidence will be derived in part from healthy AYAs as well as other AYA chronic illness populations.

\section{Exercise strategies in AYA cancer survivors - a review of the literature}

To better understand the strategies used to target improvements in exercise in AYA survivors to date, a systematic search was conducted for studies published prior to April 2015 in two databases: MEDLINE and PsycINFO. The search consisted of the subject headings and text words: "adolescent cancer survivor", "young adult cancer survivor", or "AYA cancer survivor" combined with each of the following: "health behaviors" AND "intervention"; "exercise" AND "intervention"; "physical activity" AND "intervention". All search results were limited to English language. A secondary search was conducted by manually reviewing the reference sections of identified studies and review articles. After obtaining all relevant manuscripts, abstracts were screened to ensure applicability to the topic. Manuscripts were included if they 1 ) were empirical studies reporting on an intervention designed to promote exercise or physical activity for participants either 
receiving treatment for or a survivor of cancer; 2) included adolescents between the ages of 15 and 18 years or young adults between the ages of 18 and 29 years; and 3) included a sample size of at least ten participants. Observational studies (including case reports and case-control studies) and surveys were excluded from this review.

The literature search, including primary and secondary search strategies, yielded 463 articles. Following a review of titles and abstracts and taking into account duplicate search returns, the search was narrowed to 22 papers that appeared to meet the inclusion criteria described earlier. Careful readings of the 22 full manuscripts further reduced the field to nine studies that met all inclusion criteria. ${ }^{33-42}$ Of these studies, six included adolescent survivors, two addressed young adult survivors only, and one included both young adult and adolescent survivors. Interventions were heterogeneous across study design (nonrandomized, noncontrolled versus randomized, controlled trial), sample sizes (range $=10-251$ ), ages (range $=3-39$ years of age), diagnoses (acute lymphoblastic leukemia [ALL], brain tumor, mixed diagnoses), and points of intervention (on-treatment, immediate postcompletion of treatment, long-term survivorship). See Table 1 for a description of the included studies.

Of the studies including adolescents, four included survivors only (aged 6-39 years), one included on-treatment participants only (aged 3-17 years), and one included youth diagnosed with cancer regardless of treatment status (aged 14-18 years). ${ }^{33-39}$ No adverse events or perceived risks were reported in any of the studies regardless of intervention timing or treatment status, suggesting that exercise interventions are safe and appropriate from a medical standpoint for this population.

For those studies targeting exercise in adolescent survivors, five of the six interventions showed significant improvement in outcomes related to exercise following completion of the intervention; however, no interventions evaluated outcomes based on the individual strategies comprising the intervention. ${ }^{33-37}$ All of the interventions demonstrating benefits employed two or more psychosocial strategies, including in vivo exercise training, psychoeducation, goal-setting, self-monitoring, behavioral reinforcement, or social support. Four of the interventions also involved trained interventionists to ensure participant adherence with the intervention, including a mix of supervised instruction and unsupervised exercise sessions allowing adolescents to practice learned skills at home. ${ }^{33,35-37}$ Intervention duration varied from 4 days to 24 months with no apparent association between length of intervention and positive change in exercise outcomes. While initial gains were noted in five of the six interventions, only the 4-day adventure-based exercise-promoting intervention by $\mathrm{Li}$ et al demonstrated long-term success through the maintenance of exercise, self-efficacy, and quality-of-life gains at 9-month follow-up. ${ }^{37}$ Of note, the one study that did not show improvements in exercise outcomes was comprised of a single psychosocial strategy, goal-setting, and, while activity was encouraged, strategies focused on active exercise engagement were not included. ${ }^{38,39}$

Two interventions included young adult survivors. ${ }^{40,41}$ Rabin et al conducted a 12-week Internet-based exercise intervention guided by social cognitive theory (SCT) and the transtheoretical model (TTM) of behavior change with 18 young adult cancer survivors. ${ }^{40}$ These interventions used psychoeducation, goal-setting, and self-monitoring to increase exercise via a Web-based exercise manual matched to participants' stage of readiness to engage in exercise. Feasibility and participant satisfaction were high, and self-reported outcomes indicated that exercise, mood, and fatigue were significantly improved following the intervention compared to the control group. Adherence and long-term maintenance of outcomes were not measured, limiting the ability to draw conclusions about the intervention's efficacy. Information was not available regarding the number of strategies each participant utilized during the intervention, precluding conclusions regarding the efficacy of individual intervention components.

Valle et al conducted a similar 12-week intervention with 86 young adult survivors using a Facebook-based program called FITNET aimed to increase moderate-to-vigorous exercise. ${ }^{41}$ The intervention utilized psychoeducation, goalsetting, self-monitoring, and social support to promote self-efficacy for exercise. Self-report outcome measures included exercise frequency and amount, self-efficacy for exercise, social support for exercise, and self-monitoring of exercise. Interestingly, the intervention group showed lower self-efficacy for exercise and social support from online friends compared to the control group. No gains in moderateto-vigorous exercise were found based on the intervention, though social support and self-efficacy were positively associated with moderate-to-vigorous exercise across both groups. ${ }^{41}$ Again, engagement in individual exercise-promoting strategies across the intervention was not analyzed.

Lastly, the intervention delivered to both young adults and adolescents implemented a 16-week home-based exercise program to 17 survivors of ALL between the ages of 16 and 30 years. ${ }^{42}$ The home exercise program consisted of 3-4 days per week of strength exercises, and aerobic exercise of the 


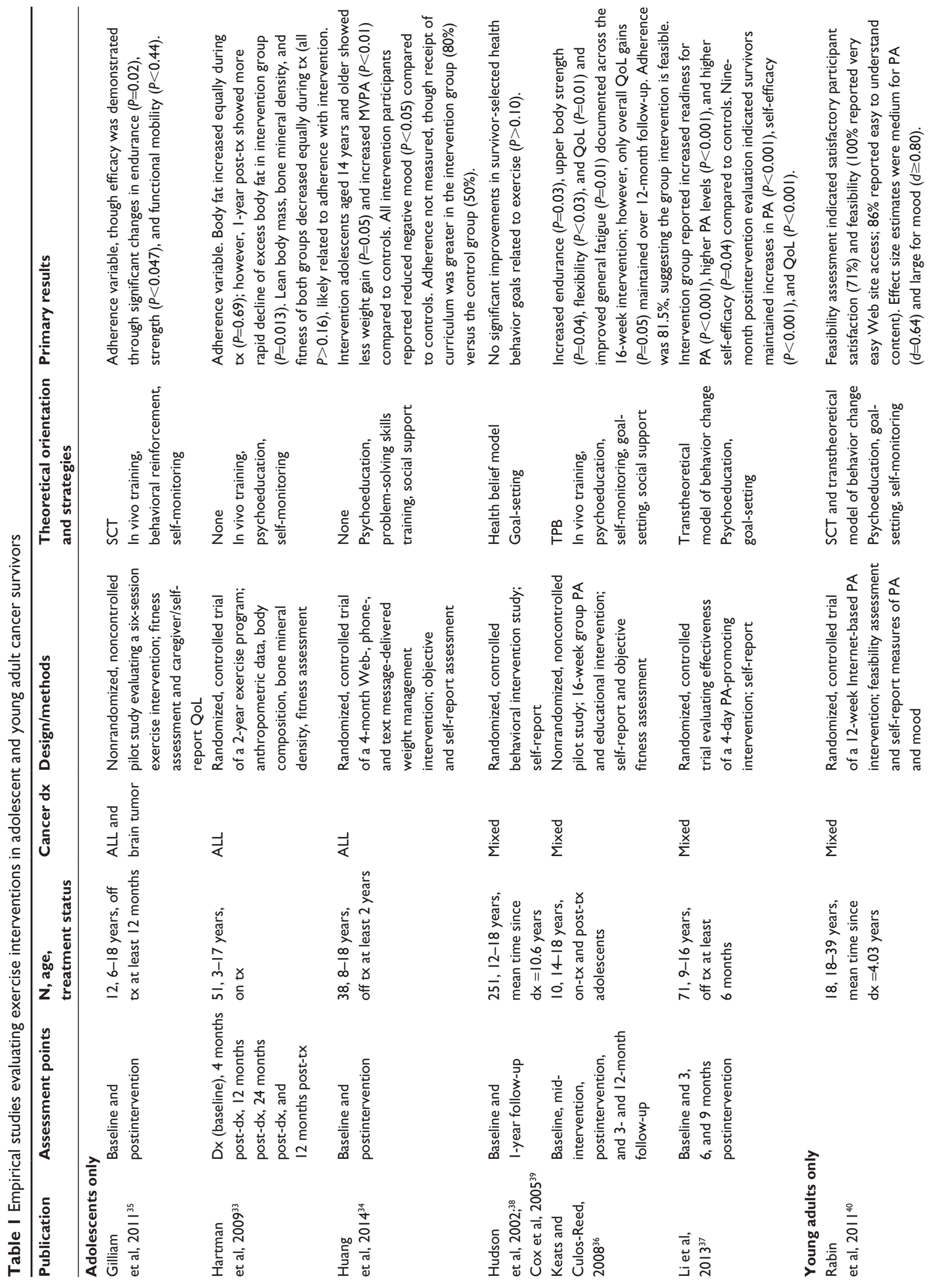



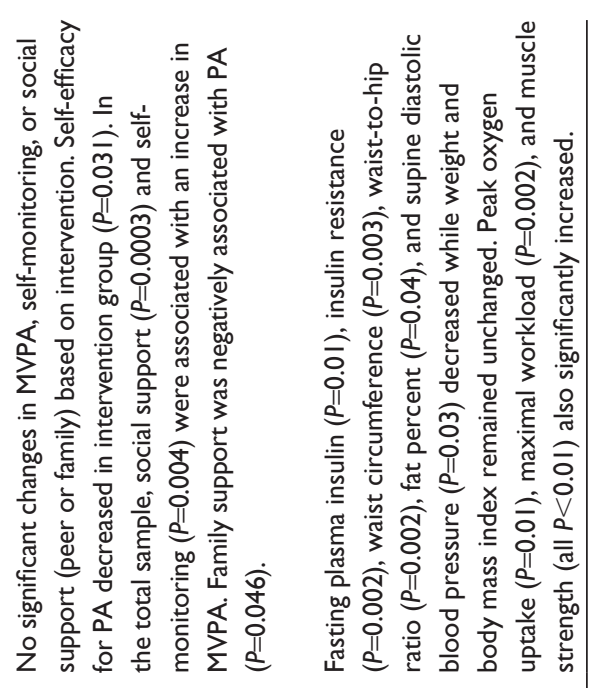

survivor's choice for 30 minutes or more three times per week was encouraged. Self-monitoring was encouraged to monitor daily engagement in exercise. Additionally, problem-solving skills training was provided by a counselor twice weekly via telephone to increase motivation and maintain survivors' engagement in the exercise program. Results indicated that immediately following the intervention, fasting insulin, insulin resistance, waist circumference, waist-to-hip ratio, percent fat, and supine diastolic blood pressure significantly decreased. These outcomes were supported by concurrent improvements in fitness, including peak oxygen uptake and maximal workload, and muscle strength (in abdominals, lower back, and leg). Long-term maintenance of physical activity and related outcomes were not assessed.

While the literature to date provides promising preliminary evidence for the feasibility and benefits of exercise interventions in AYA survivors, specific limitations of the interventions conducted thus far must be considered. First, and perhaps most importantly in the context of the current review, the individual strategies comprising the interventions varied widely, both in type, number of strategies employed, and delivery method. For example, Järvelä et al's homebased exercise intervention included self-monitoring and problem-solving skills training, as compared to Valle et al's intervention, which utilized psychoeducation, goal-setting, self-monitoring, and social support delivered via an Internetbased platform. ${ }^{41,42}$ Given the variability in type, number, and delivery method of exercise-promoting strategies, definitive conclusions guiding recommendations for duration, intensity, and modality are limited at this time.

In addition to our inability to draw causal links between exercise strategies and increased exercise engagement, additional methodological issues, including small sample sizes, variable adherence rates, the absence of long-term follow-up to assess maintenance of exercise behaviors, and the reliance on self-report measures of exercise engagement, each limit our ability to draw conclusions concerning the exercise strategies necessary to induce and sustain behavior change in AYA survivors. Ongoing research is needed and will ideally consist of randomized, controlled trials designed to evaluate the unique contribution of each intervention component.

\section{Recommendations for strategies to promote exercise in AYA survivors}

Despite the lack of conclusive evidence regarding the active elements necessary to promote and maintain adequate exercise in AYA survivors, several strategies, grounded in psychosocial research and health behavior 
theories, and expounded on in the current literature review, are offered below. First, an overview of the developmental issues unique to AYA survivors initiating behavior change is considered, followed by a brief introduction of the leading theories used to guide psychosocial strategies aimed at increasing exercise behaviors and, finally, recommendations regarding specific strategies to promote AYA exercise.

\section{Developmental issues related to AYA survivors' exercise engagement}

AYAs, regardless of medical history, face challenges as they transition from childhood to adulthood. Not only are they experiencing rapid physical, cognitive, and social changes, they are also navigating several life tasks including the development of self-identity, emotional and financial autonomy from family, peer and intimate relationships, and educational and career goals. ${ }^{43,44}$ AYA survivors must navigate these tasks while at the same time integrating the impact of their cancer diagnosis and treatment, including the emotional, social, and physical late effects manifesting from diagnosis and treatment. ${ }^{45,46}$ The dual challenges of normal adolescence and young adulthood layered with the physical and psychosocial stressors inherent to the cancer journey likely influence AYA survivors' developmental trajectories, including their establishment of healthy lifestyle behaviors such as exercise. ${ }^{47}$ Both for healthy youth and particularly AYAs, an age-related decline in physical activity begins in preadolescence and continues into adulthood. ${ }^{48-51}$ This decline appears to be steeper for AYA survivors as research indicates that AYA survivors report significantly lower levels of physical activity compared to healthy peers without a history of serious illness or injury. ${ }^{52}$ It is possible that AYA survivors are impacted doubly, with age and illness experience both playing a role. In support of this, medical provider-reported survivor health problems and ratings of cancer treatment intensity were not related to survivor physical activity, but rather survivor-reported negative beliefs about their cognitive capacity (eg, I do not learn new things as easily as other people) predicted lower physical activity 2 months later. ${ }^{52}$ This finding supports the role of cognitions in exercise engagement as documented in other studies with AYA survivors as well as the importance of implementing exercise regimens early in cancer diagnosis. ${ }^{53,54}$ It is likely that disruptions in the developmental trajectory such as the formation of self-efficacy, perceived health competencies and health beliefs, and perceived behavioral control interact with natural maturation, leading to significantly lower levels of exercise during this critical period of physical and psychological development.

\section{Theories guiding psychosocial strategies to increase exercise engagement}

To understand the potential influences on survivor exercise engagement and strategies that may increase these activities, a working knowledge of theories of health behaviors is important. In the exercise and physical activity literature for both typically developing individuals and survivors specifically, three key theories have guided interventions, including the TTM, SCT, and the theory of planned behavior (TPB), all of which generally posit that specific intrapersonal, interpersonal, or extrapersonal factors interact to promote or inhibit engagement in exercise. ${ }^{55}$ Each theory is discussed briefly in the following paragraphs.

The TTM, also known as the stages of change model, is a cyclic, dynamic model of behavior change and has been used in two of the nine interventions with AYAs. ${ }^{37,40}$ The TTM consists of five stages varying in an individual's degree of commitment to behavior change: 1) precontemplation: exercise adoption in the next 6 months is not being considered; 2) contemplation: exercise adoption in the next 6 months is being considered; 3 ) preparation: exercise patterns are not regular; 4) action: regular exercise for less than 6 months; and 5) maintenance: regular exercise for 6 months or more. Relapse is an expected process in the TTM, and reengagement of individuals can occur at any level following the decrease or cessation of a desired behavior. Strategies based on the TTM have been found to be effective at increasing exercise and fitness, reducing pain and fatigue, and improving general health. ${ }^{55,56}$

Behavioral engagement is influenced by numerous interactive antecedents, including cognitive and environmental variables that may facilitate or impede adoption of the desired behavior, as well as various positive and negative consequences that affect future engagement in the behavior. SCT was used in three of the nine interventions for AYAs ${ }^{35,40,41}$ and focuses on the importance of the individual to be able to control a desired behavior and how changes in the individual's cognitions (self-efficacy, outcome expectations) and/or the environment (social support) can produce changes in behavior and has been found to be effective in increasing exercise, improving nutritional intake, and reducing body weight and body mass. ${ }^{55}$

Finally, the TPB, used in one of the nine interventions, ${ }^{36}$ postulates that behavior is predicted by intention, which in turn is predicted by cognitions including attitude 
(positive or negative evaluation of performing a given behavior), subjective norms (perception of social influences), and perceived control (an individual's perception of the ease or difficulty of performing a particular behavior). ${ }^{57}$ Taken together, these components lead to formation of behavioral intention. As such, an individual's intention to engage in a behavior will be stronger when they hold a favorable attitude toward the behavior, believe that important others think they should perform the behavior, and believe that the behavior is under their control. Strategies based on the TPB have been applied with various adult and young adult cancer populations but have only been assessed in one intervention in newly diagnosed adult breast cancer patients, finding that perceived behavioral control was both directly and indirectly linked to exercise. The theory as a whole was not supported, as there were no direct effects between any TPB constructs and intention to exercise. ${ }^{58}$

\section{Psychosocial strategies to maximize survivor exercise engagement}

Based on the literature conducted with AYA survivors to date along with work in other chronic illness populations, interventions including behavioral, cognitive, and/or social support components may encourage initiation and maintenance of recommended exercise levels in AYA survivors. ${ }^{59}$ The recommendations put forth below are a result of the current literature review as well as the literature on healthy AYAs and AYAs with chronic illnesses with consideration of AYA developmental needs and preferences. Specific strategies are a result of one or more of the social and behavioral theories reviewed previously and allow for a theory-driven approach to individually tailored behavior change intervention.

\section{In vivo exercise training}

Three of the nine interventions reported utilizing in vivo exercise training as a component in their intervention. ${ }^{33,35,36}$ Direct instruction with modeling of targeted exercise strategies is key to the initiation of a new exercise plan. Survivors who do not have a history of exercising or physical activity engagement may not know the basics of cardiovascular and/or resistance training, thus decreasing their likelihood to engage in these activities. Direct instruction with modeling, unlike traditional education, offers the survivor the opportunity to practice exercises with immediate feedback. This allows the survivor to master exercise techniques as well as offers an opportunity to individualize exercise modalities to the survivor's individual needs, based on preferences and any physical limitations that may exist. Ideally, exercise training will involve multiple sessions with a trained professional spread out over time so that the survivor may practice exercises on their own in their natural setting, promoting identification of barriers to exercise that can be addressed in-session and creating an opportunity for problem-solving to support adherence. If possible, in an effort to quantify an observable change in body composition and provide direct evidence to the survivor of the effects of exercise, body composition assessment may be considered as part of in vivo training, thus promoting adherence and education at the same time.

\section{Psychoeducation}

Six of the nine interventions reported providing psychoeducation as a component in their intervention. . $3,34,36,37,40,41^{2}$ Psychoeducation encompasses a broad range of activities that generally combine education regarding exercise and its relation to cancer survivorship with counseling to increase the likelihood of engagement in this behavior. Though psychoeducation alone does not generally result in behavior change, it can help provide important knowledge necessary for success. Depending on the setting and program design, psychoeducation may be delivered individually or in groups and may be tailored to a specific subpopulation (eg, survivors diagnosed with ALL) or standardized to be applicable to diverse patient populations (eg, AYA survivors diagnosed with any cancer). Delivery format may include face-to-face, telephone, social media, or email, though engagement of AYAs through current technological means, including social media or smartphone applications, may be most preferred. Moreover, these interventions overcome barriers of travel and distance, which are significant for this patient population. Given that studies using psychoeducational interventions tend to vary substantially in specific content, format, frequency, and timing, it is difficult to draw conclusions regarding the unique contribution of this strategy to behavior change or intervention efficacy.

\section{Goal-setting}

Five of the nine interventions reported implementing goalsetting as a component in their intervention. ${ }^{36-41}$ Helping survivors identify and develop their own goals for exercise may increase survivor motivation, perceived control, intention to exercise, and, ultimately, commitment toward goal attainment. One of the most widely used goal-setting techniques is called the SMART approach, whereby goals are set using the following criteria: specific, measurable, attainable, relevant, and time-based. ${ }^{60}$ Given their developmental stage, AYA survivors may be more focused on the immediate 
future as opposed to long-term outcomes, so short-term goals (eg, I will be able to run in a $5 \mathrm{~K}$ race in 3 months with my college roommate) may be more appropriate and productive as opposed to long-term goals (eg, I will decrease my risk for cardiovascular disease when I am older by exercising five times a week for 30 minutes). Goal-setting will work best when used in tandem with additional strategies such as self-monitoring or social support, as research indicates it is not effective in isolation. ${ }^{59,60}$

\section{Self-monitoring}

Six of the nine interventions reported utilizing self-monitoring as a component in their intervention. ${ }^{33,35,36,40-42}$ Selfmonitoring of a desired behavior is a core component of self-regulation as it precedes and supports one's ability to evaluate whether progress has been made toward an intended goal. ${ }^{61,62}$ It requires deliberate attention to a behavior and the details of that behavior with documentation of these details over time. Research suggests that consistent self-monitors exercise more often, report less barriers to exercise, and achieve greater weight loss over time compared to those who do not engage in consistent self-monitoring of exercise. ${ }^{58,62}$ To increase adherence, self-monitoring should be limited to a single desired behavior associated with a well-defined goal. In addition, given the preference of AYAs for technology-based interventions, the use of smart devices (eg, phones, tablets, watches) may be a desirable method for tracking exercise and increasing adherence to exercise programs. ${ }^{18}$

\section{Problem-solving skills training}

Two of the nine interventions reported providing problemsolving skills training as a component in their intervention. ${ }^{34,42}$ In conjunction with self-monitoring that allows AYAs to identify patterns of exercise and barriers to engagement, problem-solving skills training to generate solutions to identified barriers is also a well-supported strategy to improve health behavior engagement in several chronic illness groups. ${ }^{63}$ Problem-solving skills training generally consists of the following components: defining the problem, generating alternative solutions, deciding on a solution, and implementing and evaluating the solution. Providing AYAs with the skills to systematically think through any barriers or issues they experience when attempting to exercise will not only allow them to work through these barriers, but it will provide them with the skills necessary to independently think through barriers that may arise in the future. Benefits of this technique are twofold as they increase the likelihood of an AYA engaging in exercise while concurrently promoting the development of survivor self-efficacy and perceived control for exercise.

\section{Motivational interviewing}

Though no studies with AYA cancer survivors have incorporated motivational interviewing (MI) to date, it has been widely used to promote adolescent health behavior engagement in both healthy and chronically ill youth. ${ }^{64-67}$ MI is defined as a collaborative, person-centered approach to help support motivation for change and is a well-supported intervention strategy for increasing exercise in both adolescents and adults. ${ }^{64-67}$ The goal of MI is to allow the AYA to become the primary advocate and agent for change through the exploration and resolution of ambivalence toward exercise. The four basic principles of MI include expressing empathy, developing discrepancy, rolling with resistance, and supporting self-efficacy. Expressing empathy requires that providers actively listen to and understand the AYA's experience without judgment, using this experience, not the provider's idea of what is needed, as the foundation for change. Developing discrepancy explores the pros and cons of both current behavior (sedentary) and new behavior (exercise) from the AYA's perspective. Highlighting discrepancies between current behaviors and future goals can help support motivation for change. Rolling with resistance involves the provider normalizing resistance and ambivalence as normal, thereby decreasing frustration and allowing for active problem-solving around perceived barriers to exercise. Lastly, supporting self-efficacy is a means for helping to promote the internalization of beliefs of control and capability to engage in exercise behaviors, thus instilling confidence in the AYA survivor that they can be successful as they work toward their exercise goal. Additional benefits of MI include that it can be implemented in relatively few sessions, it can be conducted within a busy clinic schedule (ie, only adds a few minutes to patient visits), and it does not increase costs. MI is noted to increase engagement in therapy and intention to change and is meant to be used with patients having the most difficulty with health behavior change to reduce risk. ${ }^{67,68}$

\section{Social support}

Three of the nine interventions reported implementing social support as a component in their intervention. ${ }^{34,36,41}$ Both family support and peer support for exercise are associated with exercise among healthy youth and adolescents who have survived cancer. ${ }^{69-72}$ In the transition into adolescence and young adulthood, however, research indicates that 
peer support begins to gain importance as compared to family support in the facilitation of survivor exercise and that this support is associated with moderate-to-vigorous exercise via increases in survivor self-efficacy. ${ }^{52}$ Valle et al reported similar findings, demonstrating that social support mediated the relations between AYA survivor participation in an exercise intervention and increases in moderate-tovigorous exercise. ${ }^{41}$ Incorporating peer support for AYAs may lead to greater exercise engagement through direct peer encouragement for AYA engagement in exercise, ongoing accountability related to exercise goals, and support for continued engagement in an active lifestyle. Greater success with weight loss and achievement of health goals for adolescents who are overweight has been linked to positive social support from peers as well as high ratings of self-efficacy and positive perception of family functioning. ${ }^{73}$ Given AYAs' preferences for online intervention formats, social support may be effectively delivered through technological means, including social media or smartphone applications that would allow for instantaneous and ongoing peer support. Use of technology could overcome barriers of travel and distance, which are significant for this patient population. Finally, future research is needed to determine whether peer support is most effective when coming from another AYA survivor versus from a healthy peer.

\section{Conclusion}

Despite known benefits of exercise for healthy aging and to offset late effects of cancer, AYAs are not meeting the minimum recommendations to realize these benefits. It is important for health care providers to 1) educate AYA survivors on the benefits of exercise related to health, both in typical development and in relation to their cancer journey and 2) adopt targeted, evidence-based strategies aimed at promoting survivor exercise. It is hoped that attention to these issues will provide the platform for constructive dialogue toward the development of best practice strategies to optimize exercise training in AYAs. From a research perspective, while several interventions utilizing a wide variety of psychosocial strategies to promote exercise have been successful, additional research is needed to evaluate long-term maintenance of exercise behaviors, with specific attention given to the psychosocial strategies that may support engagement in these behaviors. Interventions should be designed such that exercise-promoting strategies may be evaluated both individually and within the context of the intervention as a whole in their contribution to survivor exercise engagement. Further, measures tracking survivors' utilization of these strategies both during the active intervention period and across long-term follow-up should be implemented so that direct effects of individual strategies on physiological outcomes may be evaluated. From a clinical perspective, when adopting strategies to increase AYA survivor exercise, consideration should be given to the survivor's physical strengths and weaknesses, exercise preferences, and individual goals and/or motivations in order to maximize survivor commitment to the exercise plan. As with any behavior change, it is not realistic to expect that survivors, especially those who have led a sedentary lifestyle for an extended period of time, will reach the recommended goals for exercise immediately. Behavior change will likely require the adoption of several strategies delivered in combination and over time in order to achieve AYAs' long-term adoption of an active lifestyle. In some cases, referral to a specialist team of providers, such as a weight management clinic, may be necessary to assist with creating appropriate activity goals, monitoring progress, and increasing the intensity as needed. ${ }^{74}$

\section{Disclosure}

The authors report no conflicts of interest in this work.

\section{References}

1. Bleyer A, Viny A, Barr R. Cancer in 15- to 29-year-olds by primary site. Oncologist. 2006;11:590-601.

2. Oeffinger $\mathrm{KC}$, Tonorezos ES. The cancer is over, now what?: Understanding risk, changing outcomes. Cancer. 2011:117:2250-2257.

3. Adolescent and Young Adult Oncology Press Review Group. Closing the Gap: Research and Care Imperatives for Adolescents and Young Adults with Cancer. NIH Publication No. 06-6067. US Department of Health and Human Services, National Institutes of Health, National Cancer Institute, LIVESTRONG ${ }^{\mathrm{TM}}$ Young Adult Alliance; 2006.

4. Mertens AC, Yasui Y, Neglia JP, et al. Late mortality experience in five-year survivors of child and adolescent cancer: the Childhood Cancer Survivor Study. J Clin Oncol. 2001;19:3163-3172.

5. Fosså SD, Aass N, Harvei S, Tretli S. Increased mortality rates in young and middle-aged patients with malignant germ cell tumours. Br J Cancer. 2004;90:607-612.

6. Tai E, Buchanan N, Townsend J, Fairley T, Moore A, Richardson LC. Health status of adolescent and young adult cancer survivors. Cancer. 2012;118:4884-4891.

7. Bhatia S, Landier W, Toogood AA, Hawkins M. Adolescent and young adult cancer survivors: late effects of treatment. In: Bleyer AW, Barr RD, editors. Cancer in Adolescents and Young Adults. New York: Springer; 2007. 411-430.

8. Doyle C, Kushi LH, Byers T, et al; 2006 Nutrition, Physical Activity and Cancer Survivorship Advisory Committee; American Cancer Society. Nutrition and physical activity during and after cancer treatment: an American Cancer Society guide for informed choices. CA Cancer J Clin. 2006;56:323-353.

9. US Department of Health and Human Services. Physical Activity Guidelines for Americans. Washington: US Department of Health and Human Services; 2008.

10. Stolley MR, Restrepo J, Sharp LK. Diet and physical activity in childhood cancer survivors: a review of the literature. Ann Behav Med. 2010;39:232-249. 
11. Wolin KY, Ruiz JR, Tuchman H, Lucia A. Exercise in adult and pediatric hematological cancer survivors: an intervention review. Leukemia. 2010;24:1113-1120.

12. Huang TT, Ness KK. Exercise interventions in children with cancer: a review. Int J Pediatr. 2011;2011:461512.

13. Barnes MJ, Demark-Wahnefried W. Importance of balanced diet and physical activity during and after cancer treatment in adolescent patients. Clin Oncol Adolesc Young Adults. 2014;4:13-20.

14. Bélanger LJ, Plotnikoff RC, Clark A, Courneya KS. Physical activity and health-related quality of life in young adult cancer survivors: a Canadian provincial survey. J Cancer Surviv. 2011;5:44-53.

15. Haskell WL, Lee IM, Pate RR, et al; American College of Sports Medicine; American Heart Association. Physical activity and public health: updated recommendations for adults from the American College of Sports Medicine and the American Heart Association. Circulation. 2007;116:1081-1093.

16. Rock CL, Doyle C, Demark-Wahnefried W, et al. Nutrition and physical activity guidelines for cancer survivors. CA Cancer J Clin. 2012;62: 243-274.

17. World Health Organization. Global Recommendations on Physical Activity for Health. Geneva: World Health Organization; 2010. Available from: http://whqlibdoc.who.int/publications/2010/9789241599979_eng. pdf. Accessed April 29, 2015.

18. Badr H, Chandra J, Paxton RJ, et al. Health-related quality of life, lifestyle behaviors, and intervention preferences of survivors of childhood cancer. J Cancer Surviv. 2013;7:523-534.

19. Murnane A, Gough K, Thompson K, Holland L, Conyers R. Adolescents and young adult cancer survivors: exercise habits, quality of life and physical activity preferences. Support Care Cancer. 2015;23:501-510.

20. US Department of Health and Human Services. Physical Activity and Health: A Report of the Surgeon General. Atlanta: US Department of Health and Human Services, Centers for Disease Control and Prevention, National Center for Chronic Disease Prevention and Health Promotion; 1996.

21. Hamilton MT, Hamilton DG, Zderic TW. Role of low energy expenditure and sitting in obesity, metabolic syndrome, type 2 diabetes, and cardiovascular disease. Diabetes. 2007;56:2655-2667.

22. Katzmarzyk PT. Physical activity, sedentary behavior, and health: paradigm paralysis or paradigm shift? Diabetes. 2010;59:2717-2725.

23. Sonne MP, Alibegovic AC, Højbjerre L, Vaag A, Stallknecht B, Dela F. Effect of 10 days of bedrest on metabolic and vascular insulin action: a study in individuals at risk for type 2 diabetes. J Appl Physiol (1985). 2010;108:830-837.

24. Hamilton MT, Hamilton DG, Zderic TW. Exercise physiology versus inactivity physiology: an essential concept for understanding lipoprotein lipase regulation. Exerc Sport Sci Rev. 2004;32:161-166.

25. Højbjerre L, Sonne MP, Alibegovic AC, et al. Impact of physical inactivity on subcutaneous adipose tissue metabolism in healthy young male offspring of patients with type 2 diabetes. Diabetes. 2010;59:2790-2798.

26. Stephens BR, Granados K, Zderic TW, Hamilton MT, Braun B. Effects of 1 day of inactivity on insulin action in healthy men and women: interaction with energy intake. Metabolism. 2011;60:941-949.

27. Zderic TW, Hamilton MT. Physical inactivity amplifies the sensitivity of skeletal muscle to the lipid-induced downregulation of lipoprotein lipase activity. J Appl Physiol (1985). 2006;100:249-257.

28. Saunders TJ. The health impact of sedentary behaviour in children and youth. Appl Physiol Nutr Metab. 2014;39:402.

29. Ness KK, Krull KR, Jones KE, et al. Physiologic frailty as a sign of accelerated aging among adult survivors of childhood cancer: a report from the St Jude Lifetime Cohort study. J Clin Oncol 2013;31: 4496-4503.

30. Adamsen L, Andersen C, Midtgaard J, Møller T, Quist M, Rørth M. Struggling with cancer and treatment: young athletes recapture body control and identity through exercise: qualitative findings from a supervised group exercise program in cancer patients of mixed gender undergoing chemotherapy. Scand J Med Sci Sports. 2009;19:55-66.
31. Järvelä LS, Niinikoski H, Lähteenmäki PM, et al. Physical activity and fitness in adolescent and young adult long-term survivors of childhood acute lymphoblastic leukaemia. J Cancer Surviv. 2010; 4:339-345.

32. Sala A, Talsma D, Webber C, Posgate S, Atkinson S, Barr R. Bone mineral status after treatment of malignant lymphoma in childhood and adolescence. Euro J Cancer Care (Engl). 2007;16:373-379.

33. Hartman A, te Winkel ML, van Beek RD, et al. A randomized trial investigating an exercise program to prevent reduction of bone mineral density and impairment of motor performance during treatment for childhood acute lymphoblastic leukemia. Pediatr Blood Cancer. 2009;53:64-71

34. Huang JS, Dillon L, Terrones L, et al. Fit4Life: a weight loss intervention for children who have survived childhood leukemia. Pediatr Blood Cancer. 2014;61:894-900.

35. Gilliam MB, Ross K, Walsh A, et al. Healthy Heroes: a token economy to increase adherence with a community-based exercise intervention for childhood cancer survivors. Rehabilitation Oncology. 2011;29:16-22.

36. Keats MR, Culos-Reed SN. A community-based physical activity program for adolescents with cancer (project TREK): program feasibility and preliminary findings. J Pediatr Hematol Oncol. 2008;30: $272-280$.

37. Li HC, Chung OK, Ho KY, Chiu SY, Lopez V. Effectiveness of an integrated adventure-based training and health education program in promoting regular exercise among childhood cancer survivors. Psychooncology. 2013;22:2601-2610.

38. Hudson MM, Tyc VL, Srivastava DK, et al. Multi-component behavioral intervention to promote health protective behaviors in childhood cancer survivors: the project study. Med Pediatr Oncol. 2002;39:2-1; discussion 2.

39. Cox CL, McLaughlin RA, Rai SN, Steen BD, Hudson MM. Adolescent survivors: a secondary analysis of a clinical trial targeting behavior change. Pediatr Blood Cancer. 2005;45:144-154.

40. Rabin C, Dunsiger S, Ness KK, Marcus BH. Internet-based physical activity intervention targeting young adult cancer survivors. J Adolesc Young Adult Oncol. 2011;1:188-194.

41. Valle CG, Tate DF, Mayer DK, Allicock M, Cai J. Exploring mediators of physical activity in young adult cancer survivors: evidence from a randomized trial of a Facebook-based physical activity intervention. J Adolesc Young Adult Oncol. 2015;4:26-33.

42. Järvelä LS, Kemppainen J, Niinikoski H, et al. Effects of a home-based exercise program on metabolic risk factors and fitness in long-term survivors of childhood acute lymphoblastic leukemia. Pediatr Blood Cancer. 2012;59:155-160.

43. Harter S. The Construction of the Self: A Developmental Perspective. New York: The Guilford Press; 1999.

44. Ingersoll G. Adolescence. 2nd ed. Englewood Cliffs: Prentice Hall; 1989.

45. Evan EE, Zeltzer LK. Psychosocial dimensions of cancer in adolescents and young adults. Cancer. 2006;107:1663-1671.

46. Love C, Sabiston CM. Exploring the links between physical activity and posttraumatic growth in young adult cancer survivors. Psychooncology. 2011;20:278-286.

47. Takei Y, Akiko O, Ozawa M, et al. Psychosocial difficulties in adolescent and young adult survivors of childhood cancer. Pediatr Int. 2015;57:239-246

48. Arroyave WD, Clipp EC, Miller PE, et al. Childhood cancer survivors' perceived barriers to improving exercise and dietary behaviors. Oncol Nurs Forum. 2008;35:121-130.

49. Ness KK, Leisenring WM, Huang S, et al. Predictors of inactive lifestyle among adult survivors of childhood cancer: a report from the Childhood Cancer Survivor Study. Cancer. 2009;115:1984-1994.

50. Sallis JF, Taylor WC, Dowda M, Freedson PS, Pate RR. Correlates of vigorous physical activity for children in grades 1 through 12 : comparing parent-reported and objectively measured physical activity. Pediatr Exerc Sci. 2002;14:30-44. 
51. Tyc VL, Hadley W, Crockett G. Prediction of health behaviors in pediatric cancer survivors. Med Pediatr Oncol. 2001;37:42-46.

52. Hocking MC, Schwartz LA, Hobbie WL, et al. Prospectively examining physical activity in young adult survivors of childhood cancer and healthy controls. Pediatr Blood Cancer. 2013;60:309-315.

53. Gilliam MB, Madan-Swain A, Whelan K, et al. Cognitive influences as mediators of family and peer support for pediatric cancer survivors' physical activity. Psychooncology. 2013;22:1361-1368.

54. Finnegan L, Wilkie DJ, Wilbur J, Campbell RT, Zong S, Katula S. Correlates of physical activity in young adult survivors of childhood cancers. Oncol Nurs Forum. 2007;34:E60-E69.

55. Pinto BM, Floyd A. Theories underlying health promotion interventions among cancer survivors. Sem Oncol Nurs. 2008;24:153-163.

56. Gourlan M, Sarrazin P, Trouilloud D. Motivational interviewing as a way to promote physical activity in obese adolescents: a randomisedcontrolled trial using self-determination theory as an explanatory framework. Psychol Health. 2013;28:1265-1286.

57. Ajzen I. The theory of planned behavior. Organ Behav Hum Decis Process. 1991;50:179-211.

58. Jones LW, Courneya KS, Fairey AS, Mackey JR. Effects of an oncologist's recommendation to exercise on self-reported exercise behavior in newly diagnosed breast cancer survivors: a single-blind, randomized controlled trial. Ann Behav Med. 2004;28:105-113.

59. Raynor HA. Evidence-based treatments for childhood obesity. In: Jelalian E, Steele RG, editors. Handbook of Childhood and Adolescent Obesity. New York: Springer Science + Business Media, LLC; 2008:201-217.

60. Siegert RJ, Taylor WJ. Theoretical aspects of goal-setting and motivation in rehabilitation. Disabil Rehabil. 2004;26:1-8.

61. Burke LE, Wang J, Sevick MA. Self-monitoring in weight loss: a systematic review of the literature. J Am Diet Assoc 2011;11:92-102.

62. Steeves JA, Thompson DL, Bassett DR, Fitzhugh EC, Raynor HA. A review of different behavior modification strategies designed to reduce sedentary screen behaviors in children. J Obes. 2012;2012:379215.

63. Carels RA, Darby LA, Rydin S, Douglass OM, Cacciapaglia HM, O'Brien WH. The relationship between self-monitoring, outcome expectancies, difficulties with eating and exercise, and physical activity and weight loss treatment outcomes. Ann Behav Med. 2005;30 $182-190$
64. Woods K, Mayes S, Bartley E, Fedele D, Ryan J. An evaluation of psychosocial outcomes for children and adolescents attending a summer camp for youth with chronic illness. Child Health Care. 2013;42:85-98.

65. Miller WR, Rollnick S. Motivational Interviewing: Preparing People for Change. 2nd ed. New York: The Guilford Press; 2002.

66. Cushing CC, Jensen CD, Miller MB, Leffingwell TR. Meta-analysis of motivational interviewing for adolescent health behavior: efficacy beyond substance use. J Consult Clin Psychol. 2014;82:1212-1218.

67. Martins RK, McNeil DW. Review of motivational interviewing in promoting health behaviors. Clin Psychol Rev. 2009;29:283-293.

68. Gayes LA, Steele RG. A meta-analysis of motivational interviewing interventions for pediatric health behavior change. J Consult Clin Psychol. 2014;82:521-535.

69. Hamrin V, McGuinness TM. Motivational interviewing: a tool for increasing psychotropic medication adherence for youth. $J$ Psychosoc Nurs Ment Health Serv. 2013;51:15-18.

70. Gilliam MB, Madan-Swain A, Whelan K, Tucker DC, DemarkWahnefried W, Schwebel DC. Social, demographic, and medical influences on physical activity in child and adolescent cancer survivors. J Pediatr Psychol. 2012;37:198-208.

71. Gustafson SL, Rhodes RE. Parental correlates of physical activity in children and early adolescents. Sports Med. 2006;36:79-97.

72. Whitt-Glover MC, Taylor WC, Floyd MF, Yore MM, Yancey AK, Matthews CE. Disparities in physical activity and sedentary behaviors among US children and adolescents: prevalence, correlates, and intervention implications. J Public Health Policy. 2009;30 Suppl 1: S309-S334.

73. Van Der Horst K, Paw MJ, Twisk JW, Van Mechelen W. A brief review on correlates of physical activity and sedentariness in youth. Med Sci Sports Exerc. 2007;39:1241-1250.

74. Sampat S, Kirschenbaum DS, Gierut KJ, Germann JN, Krawczyk R. Ya gotta have friends: social support and self-efficacy predict success following immersion treatment. Obesity (Silver Spring). 2014;22: 2579-2585.
Clinical Oncology in Adolescents and Young Adults

\section{Publish your work in this journal}

Clinical Oncology in Adolescents and Young Adults is an international, peer-reviewed, open access journal publishing original research, reports, editorials, reviews and commentaries on all aspects of epidemiology, diagnosis and treatment of cancers in adolescents and young adults. The manuscript management system is completely

\section{Dovepress}

online and includes a very quick and fair peer-review system. Visit http://www.dovepress.com/testimonials.php to read real quotes from published authors. 